\title{
FLOW FIELDS ASSOCIATED WITH IN SITU MINERAL LEACHING
}

\author{
LAWRENCE K. FORBES, ANTHONY M. WATTS and GRAEME A. CHANDLER ${ }^{1}$
}

(Received 21 April 1992; revised 22 October 1992)

\begin{abstract}
A simple model for underground mineral leaching is considered, in which liquor is injected into the rock at one point and retrieved from the rock by being pumped out at another point. In its passage through the rock, the liquor dissolves some of the ore of interest, and this is therefore recovered in solution. When the injection and recovery points lie on a vertical line, the region of wetted rock forms an axi-symmetric plume, the surface of which is a free boundary. We present an accurate numerical method for the solution of the problem, and obtain estimates for the maximum possible recovery rate of the liquor, as a fraction of the injected flow rate. Limiting cases are discussed, and other geometries for fluid recovery are considered.
\end{abstract}

\section{Introduction}

The process of obtaining minerals from the ground normally involves excavation, either using an open-cut mine or else by means of subterranean tunnels. Once the mineral-bearing rock is brought to the surface, it may then be crushed, and the ore extracted from it chemically.

An alternative mining strategy, known as in situ leaching, might also be considered in instances where the grade of ore is low, or excavation is not practical. In this process, a series of injection and recovery holes is drilled into the rock. A fluid bearing an appropriate chemical such as acid (or perhaps ferric chloride) or bacteria is pumped into the injection points, and allowed to permeate the rock. As the fluid passes through the rock, the desired minerals are dissolved, and are therefore obtained in solution when the fluid is pumped out through the recovery holes. A further description of this process may be found in Friedel [3], for example, and the references therein.

Modelling the leaching process clearly poses many difficulties. Perhaps the most significant of these is that the geology of the intended site may be very complicated,

\footnotetext{
${ }^{1}$ Centre for Industrial and Applied Mathematics and Parallel Computing (CIAMP), Department of Mathematics, University of Queensland, St. Lucia, Queensland, 4072, Australia

(C) Australian Mathematical Society, 1994, Serial-fee code 0334-2700/94
} 
and perhaps not well understood. The density and porosity of the rock and the distribution of the mineral of interest would in general vary with position, and as the leaching fluid is introduced into the ore, there may be large regions formed in which the rock is incompletely saturated. Fissures may also be present, and water may flow quickly along them instead of penetrating the rock. In practice, the pressure at which the water is injected needs to be controlled to avoid opening these fissures, so as to maximize flow in the mineral bearing rock. The portions of the rock into which the leaching liquor seeps could also be affected by stratification.

In this preliminary investigation of the process of mineral leaching, we make a series of assumptions that allow us to work with the simplest possible model of this type of mining. More realistic representations of the underlying geology will be considered in future articles. We suppose here that the rock is homogeneous, and so its porosity and density are simple constants, independent of position. The fluid seeps into the rock under the influence of pressure gradients and gravity, and we assume that the flow field is not significantly affected by the chemical reactions associated with the leaching process. In reality, these reactions may be exothermic, so that a more complete model would need to include the effects of temperature variability, and perhaps also allow for regions of incomplete saturation within the inundated rock, but neither of these phenomena are included here. The distribution of fluid in the ore is therefore assumed to be governed by Darcy's law at the macroscopic level, following Lucas, Blake and Kucera [7] who give a more complete discussion of the limitations of this law relating seepage rates to pressure gradients.

For simplicity, we will consider in detail the case in which fluid is injected into the rock at one point and pumped out from another point which is either directly above or below the first point. In view of the foregoing assumptions, this ensures that the geometry of the wetted rock is axi-symmetric, which permits a simplification of the mathematical model and its numerical solution. The situation being modelled here is one in which the flow is steady, which means that there is no variation with time in the solution behaviour. Lucas, Blake and Kucera [7] point out that there may in practice be certain advantages to withdrawing fluid in an unsteady manner, but this possibility is not considered here. It will therefore be seen that the wetted region of rock forms a vertical axi-symmetric plume, but the position of the boundary of the wetted region is unknown in advance. This is therefore a free-boundary problem, and we assume a sharp interface between the (perfectly saturated) wetted rock and the rest of the ore, which is taken to be dry. In reality, there is a boundary layer of partially saturated rock between the wet and dry regions. Because the diffusivity decreases rapidly as the concentration of fluid falls below saturation, this boundary layer is sufficiently thin that its presence can be ignored. The boundary conditions at the edge of the wetted plume do not permit overhanging portions of the boundary if the assumption of total saturation within the wetted region is to be maintained, as will be seen in Section 2, 
and thus the plume is assumed to extend infinitely deeply within the rock.

The assumptions of steady flow of an incompressible fluid subject to Darcy's law lead simply to Laplace's equation for the pressure head, or "modified pressure" of Lucas, Blake and Kucera [7]. Nevertheless, the problem is still highly nonlinear, because the location of the boundary of the wetted region is unknown in advance. This free-boundary problem is thus of the withdrawal type, and is related to the work of Tuck and Vanden-Broeck [11], Hocking [4] and Forbes and Hocking [2], for example. However, the present problem involves very different geometry from that considered in the literature on withdrawal problems, since here the fluid forms a vertical plume, with nett outflow at infinity, rather than the stagnant fluid infinitely far away that is usually assumed in reservoir withdrawal simulations [11].

Previous work on mineral leaching assumes that a two-dimensional flow field is established (see Schmidt, Behnke and Friedel [9]). This allows the problem to be solved simply by a one-dimensional boundary integral equation method derived from a complex variable formulation. Further details of this approach are given by Strack [10]. The present paper extends this work to three-dimensional axi-symmetric flow fields.

Levine and Yang [6] have investigated the shape of an infinitely long bubble rising in a tube, and the geometry of their problem is similar in many respects to that of the present problem. They formulated their problem in terms of a nonlinear integral equation involving only the dependent variables on the unknown free boundary, and we also adopt an integral equation approach for the solution of the present problem, as is discussed in Section 2. However, Levine and Yang used the Stokes streamfunction as their dependent variable, with the result that the Green function required for their integral equation involves an infinite series of Bessel functions, and its numerical evaluation is a task of some considerable difficulty. By contrast, we write an integral equation for a velocity potential (modified pressure), which has the advantage that the simple point source solution may be used as the Green function. The kernel of our integral equation is therefore a relatively simple function which is quite straightforward to evaluate; it involves complete elliptic integrals as a result of the axially symmetric geometry of the problem, as is discussed by Miksis, Vanden-Broeck and Keller [8] and Forbes and Hocking [2].

The numerical method is briefly reviewed in Section 3, and in Section 4 we discuss in detail the results obtained for the simple case of a point source and a point sink of fluid lying on a vertical line. Maximum fluid withdrawal rates through the sink are computed, and the limiting cases are discussed. In Section 5, we investigate briefly the effect of replacing the point sink with a continuous circular line sink of some finite radius, and we demonstrate that increased fluid recovery rates are possible by altering the method of fluid recovery in this way. The paper concludes in Section 6, with a discussion and an indication of the directions of future research. 


\section{Mathematical formulation}

Consider a porous rock of uniform density and permeability, which extends to infinite depth. Locate a cartesian coordinate system on the rock, such that the $x-y$ plane lies along the horizontal ground and the $z$-axis points vertically. The rock thus occupies the entire lower half region.

Suppose now that holes are drilled into the rock, to enable fluid to be injected at the point $(0,0,-H)$ and recovered at the point $(0,0,-H-L)$, as in Figure 1 . The fluid will be introduced at the first point at the volume rate $Q_{1}$ (litres/second), and withdrawn from the second point at some different rate $Q_{2}$. The fluid has constant density $\rho$, and is subject to the downward acceleration $g$ of gravity. If the vector $q$ denotes the seepage velocity of the fluid through the substrate, then mass conservation gives rise to the equation

$$
\nabla \cdot \mathbf{q}=0,
$$

assuming that the wetted portion of the rock is totally saturated. For this steady problem, the seepage velocity $q$ is related to the pressure $p$ in the fluid by Darcy's law

$$
\mathbf{q}=-D \nabla(p+\rho g z),
$$

in which $D$ represents the diffusivity coefficient for the fluid through the rock. We shall assume that the properties of the rock are homogeneous, so that $D$ is a simple constant.

The form of Darcy's law (2.2) suggests the introduction of a modified pressure

$$
\Phi=p+\rho g z,
$$

following Lucas, Blake and Kucera [7]. Combining (2.1) and (2.2) then simply gives Laplace's equation to be solved for $\Phi$, which thus acts as a velocity potential.

It follows from our assumptions that there will be a vertical axi-symmetric plume of wetted rock, as in Figure 1, with a sharp interface between the perfectly saturated region inside the plume and the perfectly dry region outside. It is therefore appropriate to introduce cylindrical polar coordinates $(r, \theta, z)$ with the $z$-axis pointing vertically as before, and $r$ and $\theta$ defined through the usual relations $x=r \cos \theta$ and $y=r \sin \theta$. If the surface of the wetted rock is given by $r=\zeta(z)$, the kinematic constraint that there be no flow through this surface gives rise to the condition

$$
\frac{\partial \Phi}{\partial r}=\frac{\partial \Phi}{\partial z} \frac{d \zeta}{d z} \quad \text { on } r=\zeta(z)
$$

and the dynamic free-surface condition is simply

$$
p=0 \quad \text { on } r=\zeta(z),
$$




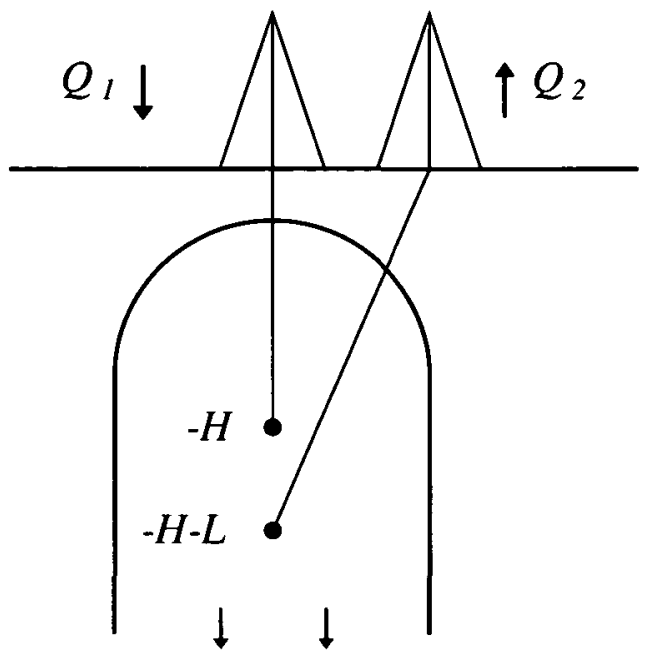

FIGURE 1. Schematic diagram, showing the injection and recovery of leaching fluid and the wetted region of rock.

in view of the assumption that the rock outside the wetted plume is totally dry.

It is necessary now to consider the flow near the injection and extraction points. In reality, fluid is forced into the rock and removed from it through pipes of small but finite radius. Nevertheless, we employ the argument proposed by Lucas, Blake and Kucera [7] that the flow a short distance away from the end of the pipe would essentially be unchanged if the pipe were replaced by a mathematical point singularity creating or annihilating a known volume of fluid per unit time. The injection point is therefore modelled by a point sink creating volume flux $Q_{1}$, located at the point $(x, y, z)=(0,0,-H)$. We have

$$
\mathbf{q}=-D \nabla \Phi \rightarrow \frac{Q_{1}[x \mathbf{i}+y \mathbf{j}+(z+H) \mathbf{k}]}{4 \pi\left[x^{2}+y^{2}+(z+H)^{2}\right]^{3 / 2}} \quad \text { as }(x, y, z) \rightarrow(0,0,-H)
$$

and hence

$$
\Phi \rightarrow \frac{Q_{1}}{4 \pi D\left[x^{2}+y^{2}+(z+H)^{2}\right]^{1 / 2}} \quad \text { as }(x, y, z) \rightarrow(0,0,-H) .
$$

The extraction point is similarly modelled by a point sink which removes fluid at the volume rate $Q_{2}$, and it creates the singularity in modified pressure given by

$$
\Phi \rightarrow-\frac{Q_{2}}{4 \pi D\left[x^{2}+y^{2}+(z+H+L)^{2}\right]^{1 / 2}} \quad \text { as }(x, y, z) \rightarrow(0,0,-H-L) .
$$


At infinite depth within the wetted plume, the fluid pressure $p$ drops to zero, and so Darcy's law (2.2) shows that the seepage speed eventually becomes $D \rho g$ in the negative $z$-direction, as $z \rightarrow-\infty$. If the radius of the plume at infinity is $R_{\infty}$, then equating the volume flow rate predicted by Darcy's law with the rate $Q_{1}-Q_{2}$ required for mass to be conserved gives the asymptotic plume radius

$$
\zeta(z) \rightarrow R_{\infty}=\left[\frac{Q_{1}-Q_{2}}{\pi \rho g D}\right]^{1 / 2} \quad \text { as } z \rightarrow-\infty .
$$

Equations (2.1)-(2.8) represent a complete statement of the problem to be solved. Before proceeding, however, we first introduce dimensionless variables, and these will be used for the remainder of this article. All lengths in the problem are scaled with respect to the sink submergence depth $H$, and the pressures $p$ and $\Phi$ are referred to the quantity $Q_{1} /(D H)$. The dimensionless form of (2.3) is therefore

$$
\Phi=p+z / F,
$$

where we have defined the parameter

$$
F=Q_{1} /\left(\rho g H^{2} D\right)
$$

which is similar to the constant introduced by Lucas, Blake and Kucera [7]. In terms of the dimensionless modified pressure $\Phi$, the dynamic boundary condition (2.5) becomes

$$
F \Phi=z \quad \text { on } r=\zeta(z) .
$$

Near the point source, (2.6) gives rise to the nondimensional form

$$
\Phi \rightarrow \frac{1}{4 \pi\left[r^{2}+(z+1)^{2}\right]^{1 / 2}} \quad \text { as }(r, z) \rightarrow(0,-1)
$$

in polar coordinates, and (2.7) yields

$$
\Phi \rightarrow-\frac{\gamma}{4 \pi\left[r^{2}+(z+1+\lambda)^{2}\right]^{1 / 2}} \quad \text { as }(r, z) \rightarrow(0,-1-\lambda)
$$

for the sink potential. Here we have introduced the dimensionless submergence depth of the sink relative to the source $\lambda=L / H$, and the fluid recovery ratio $\gamma=Q_{2} / Q_{1}$. For a steady problem, it is clearly the case that $\gamma \leq 1$, and the determination of the maximum achievable value of $\gamma$ is a major aim of the present study. In nondimensional variables, (2.8) and (2.9) at infinite submergence depth become

$$
\Phi \rightarrow \frac{z}{F}, \quad \zeta(z) \rightarrow\left[\frac{F(1-\gamma)}{\pi}\right]^{1 / 2} \quad \text { as } z \rightarrow-\infty .
$$


We remark that the choice of three dimensionless parameters $F, \lambda$ and $\gamma$ is strictly redundant since, by choosing the origin to be at the position of the injection point and using a length scale other than $H$ as reference, the number of independent parameters could have been reduced to two. In other words, the source submergence depth $H$ plays no formal role in the dimensional equations (2.1)-(2.8). Nevertheless, we have chosen to retain the three parameters because this permits us to determine the maximum permissible value of the input volume flow rate $Q_{1}$ before the top of the plume reaches the ground level. This is expected to be of importance in practice, since it is obviously desirable to avoid flooding the surface of the ground with caustic leaching fluid.

Since the modified pressure $\Phi$ satisfies Laplace's equation within the wetted region sketched in Figure 1, an integral-equation technique is available for the solution of the problem. Consider the volume $V$ shown in Figure 2. It consists of a portion of the wetted plume, and its surface $\partial V$ may be written as

$$
\partial V=S_{E}+S_{B}+S_{Q}+S_{1}+S_{1+\lambda}
$$

in which $S_{E}$ is the actual interface between the wet and dry rock with a small circle about the point $Q$ deleted, and $S_{B}$ is a bounding lower surface which will eventually be allowed to move down to $z \rightarrow-\infty$. A small hemispherical surface $S_{Q}$ is centred at the point $Q$, the source point $(0,0,-1)$ has been excluded by means of the spherical surface $S_{1}$, and a similar spherical surface $S_{1+\lambda}$ centred at the sink point excludes this singularity from the flow. It follows from Green's second formula that $\Phi$ must satisfy the equation

$$
\iint_{\partial V}\left[\Phi(P) \frac{\partial}{\partial n_{P}}\left(\frac{1}{R_{P Q}}\right)-\frac{1}{R_{P Q}} \frac{\partial \Phi(P)}{\partial n_{P}}\right] d S_{P}=0,
$$

in which $P$ denotes any point on the total surface $\partial V$. Here, $n_{P}$ denotes the outwardlydirected normal to $\partial V$ at the point $P$, and the function

$$
\begin{aligned}
1 / R_{P Q} & =1 /\left[\left(x_{Q}-x_{P}\right)^{2}+\left(y_{Q}-y_{P}\right)^{2}+\left(z_{Q}-z_{P}\right)^{2}\right]^{1 / 2} \\
& =1 /\left[r_{Q}^{2}+r_{P}^{2}-2 r_{Q} r_{P} \cos \left(\theta_{Q}-\theta_{P}\right)+\left(z_{Q}-z_{P}\right)^{2}\right]^{1 / 2},
\end{aligned}
$$

which is the inverse of the distance between points $P$ and $Q$, is harmonic within volume $V$.

It is straightforward to show that the contribution from the bottom surface $S_{B}$ becomes zero as this surface is allowed to move to infinite depth in the wetted plume. As the radius of the surface $S_{Q}$ is made to shrink to zero, the portion of the integral (2.14) due to this component $S_{Q}$ becomes simply $2 \pi \Phi(Q)$. The two spherical surfaces $S_{1}$ and $S_{1+\lambda}$, centred at the source and sink points respectively, give contributions

$$
-1 / \sqrt{r_{Q}^{2}+\left(z_{Q}+1\right)^{2}} \text { and } \gamma / \sqrt{r_{Q}^{2}+\left(z_{Q}+1+\lambda\right)^{2}}
$$




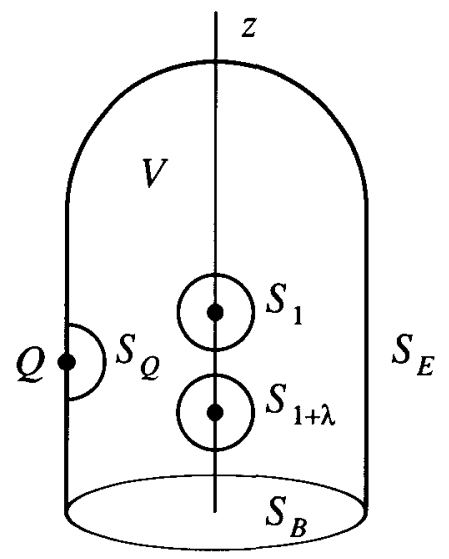

FIGURE 2. The volume of wetted rock and its bounding surfaces, used in the derivation of the integral equation.

as the radii of these two surfaces is allowed to become zero, as a consequence of (2.11) and (2.12). The relation (2.14) therefore becomes

$$
\begin{aligned}
2 \pi \Phi(Q)= & \frac{1}{\sqrt{r_{Q}^{2}+\left(z_{Q}+1\right)^{2}}}-\frac{\gamma}{\sqrt{r_{Q}^{2}+\left(z_{Q}+1+\lambda\right)^{2}}} \\
& -\iint_{S_{E}} \Phi(P) \frac{\partial}{\partial n_{P}}\left(\frac{1}{R_{P Q}}\right) d S_{P}
\end{aligned}
$$

in which $1 / R_{P Q}$ is the potential function defined in (2.15). Notice that the kinematic surface condition (2.4) has already been used to eliminate the term involving the normal derivative of $\Phi$ in the integrand on the right-hand side of this expression.

Equation (2.16) is a singular integral equation for $\Phi$, which automatically satisfies the field equation (Laplace's equation) in the wetted region of rock, in addition to the surface condition (2.4) and the two requirements (2.11) and (2.12) at the source and sink. It is possible to remove the singularity from this expression, by making use of the formula

$$
\iint_{\partial V} \frac{\partial}{\partial n_{P}}\left(\frac{1}{R_{P Q}}\right) d S_{P}=0,
$$

which follows by integrating the Laplacian of the function $1 / R_{P Q}$ over the volume $V$ in Figure 2 and then using the divergence theorem. As before, we estimate the contributions to this integral from each of the surfaces in Figure 2. The three surfaces $S_{B}, S_{1}$ and $S_{1+\lambda}$ each contribute nothing, whereas the hemisphere $S_{Q}$ produces a flux 
$2 \pi$ as its radius shrinks to zero. We therefore find that

$$
\iint_{S_{E}} \frac{\partial}{\partial n_{P}}\left(\frac{1}{R_{P Q}}\right) d S_{P}=-2 \pi
$$

and so by adding and subtracting $\Phi(Q)$ to the term $\Phi(P)$ in the integrand of (2.16), we may obtain the nonsingular integral equation

$$
\begin{aligned}
0= & \frac{1}{\sqrt{r_{Q}^{2}+\left(z_{Q}+1\right)^{2}}}-\frac{\gamma}{\sqrt{r_{Q}^{2}+\left(z_{Q}+1+\lambda\right)^{2}}} \\
& -\iint_{S_{E}}[\Phi(P)-\Phi(Q)] \frac{\partial}{\partial n_{P}}\left(\frac{1}{R_{P Q}}\right) d S_{P} .
\end{aligned}
$$

This is to be solved for the function $\Phi$ on the surface of the wetted region, subject to (2.10) and the limiting forms (2.13) deep within the rock. It may be verified that the integrand in (2.18) is indeed nonsingular, using Taylor-series expansions in the manner described by Landweber and Macagno [5].

The numerical solution of this nonlinear problem is facilitated by making use of the arclength formulation of Forbes [1]. If $s$ denotes the length along the free boundary from the top of the wetted region to the point $Q$, then the surface point $\left(r_{Q}, \theta_{Q}, z_{Q}\right)$ in axi-symmetric geometry is represented parametrically as $(r(s), z(s))$, where

$$
\left[r^{\prime}(s)\right]^{2}+\left[z^{\prime}(s)\right]^{2}=1
$$

At the top of the wetted plume,

$$
r(0)=0, \quad r^{\prime}(0)=1, \quad z^{\prime}(0)=0,
$$

although the value of $z(0)$ is unknown and must be determined as part of the solution process. Following Forbes and Hocking [2], a surface modified pressure $\phi$ is defined according to

$$
\phi(s)=\Phi(\zeta(z), z)
$$

and the dynamic boundary condition (2.10) is written in an obvious way in terms of this new variable. As a consequence of the axi-symmetric geometry, the integral equation (2.18) takes the final form

$$
\begin{aligned}
0= & \frac{1}{\sqrt{r^{2}(s)+(z(s)+1)^{2}}}-\frac{\gamma}{\sqrt{r^{2}(s)+(z(s)+1+\lambda)^{2}}} \\
& -\int_{0}^{\infty}[\phi(\sigma)-\phi(s)] \mathbb{K}(A, B, C, D) d \sigma,
\end{aligned}
$$


where the kernel function $\mathbb{K}$ may be written

$$
\mathbb{K}(A, B, C, D)=\frac{4 r(\sigma)}{D \sqrt{C+D}}\left[B K\left(\frac{2 D}{C+D}\right)+\frac{A D-B C}{C-D} E\left(\frac{2 D}{C+D}\right)\right]
$$

in which the functions $K$ and $E$ are complete elliptic integrals of the first and second kind, respectively, and the four arguments of the kernel are defined as

$$
\begin{aligned}
& A=r(\sigma) z^{\prime}(\sigma)-r^{\prime}(\sigma)(z(\sigma)-z(s)), \\
& B=r(s) z^{\prime}(\sigma), \\
& C=r^{2}(\sigma)+r^{2}(s)+(z(\sigma)-z(s))^{2}, \\
& D=2 r(s) r(\sigma) .
\end{aligned}
$$

A complete derivation of (2.21a-c) has been given by Forbes and Hocking [2], and is thus omitted here in the interests of brevity.

A complete statement of the problem is therefore provided by the integral equation (2.21), coupled with the surface condition (2.10), the asymptotic conditions (2.13), the arclength condition (2.19) and the requirements (2.20) at the top of the wetted plume. These nonlinear equations are to be solved for the interface shape $r(s)$ and $z(s)$, along with the surface modified pressure function $\phi(s)$.

We conclude this section by showing that, in steady flow in which it is assumed that the wetted portions of rock remain totally saturated, it is not possible for the interface $(r(s), z(s))$ to possess an overhanging section. In other words, the function $r=\zeta(z)$ must decrease monotonically until it attains the value zero at the top of the plume.

To see that this is true, assume the converse; that is, suppose that there is in fact a region in which the surface overhangs. On the underside of this overhanging region, it follows that $\partial z / \partial n<0$, where $n$ denotes the outward normal to the interface, as before. From (2.9), we have

$$
\frac{\partial \Phi}{\partial n}=\frac{\partial p}{\partial n}+\frac{1}{F} \frac{\partial z}{\partial n}
$$

but the kinematic condition (2.4) shows that $\partial \Phi / \partial n=0$, from which it is necessarily the case that $\partial p / \partial n>0$. Just inside the wetted region of rock near the underside of the overhanging portion, the pressure $p$ therefore increases as the interface is approached. However, (2.5) shows that pressure $p$ is zero at the interface, which means that it must be negative just inside the wetted region, and this is not possible if the rock is to remain fully saturated. Therefore, overhanging sections of the wetted plume are not permitted.

By making use of the arclength condition (2.19), it may be shown that

$$
\frac{\partial p}{\partial n}=-\frac{r^{\prime}(s)}{F}
$$


and so the necessary condition for a feasible solution to the present model is that

$$
r^{\prime}(s)>0
$$

on the interface between the wetted and the dry rock.

\section{The Numerical Solution}

In this section, we briefly describe the numerical method used for the solution of this nonlinear free-boundary problem. Possibly the greatest difficulty to be overcome is the fact that the wetted plume is of infinite extent, with nonzero outflow at infinity, and the effect of this infinite tail must be represented accurately in the numerical scheme.

A solution is sought at discrete grid points $s_{1}, s_{2}, \ldots, s_{N}$ at which the dependent variables are represented by sets of point values $\phi_{1}, \phi_{2}, \ldots, \phi_{N}$, and so on. Here, $s_{1}=0$ and the value $s_{N}$ is taken appropriately large. Before numerical solution can proceed, however, some asymptotic approximation to the flow beyond the last value $s_{N}$ of arclength must be given. On the basis of the far-field conditions (2.13), we therefore assume that

$$
\begin{array}{cc}
r(\sigma) \approx \sqrt{F(1-\gamma) / \pi}, & z(\sigma) \approx z\left(s_{N}\right)-\left(\sigma-s_{N}\right), \\
\phi(\sigma)=z(\sigma) / F & \text { for } \sigma>s_{N} .
\end{array}
$$

These approximate relations (3.1) are used to estimate the infinite tail in the integral in (2.21a), and after some calculation, we obtain

$$
\int_{s_{N}}^{\infty}[\phi(\sigma)-\phi(s)] \mathbb{K}(A, B, C, D) d \sigma \approx \mathbb{L}(T, V, W)
$$

in which

$$
\mathbb{L}(T, V, W)=\frac{2\left(2 T^{2}-V\right)}{F \sqrt{V+W}} K\left(\frac{2 W}{V+W}\right)+\frac{2 \sqrt{V+W}}{F} E\left(\frac{2 W}{V+W}\right) .
$$

As before, the functions $K$ and $E$ in this expression are complete elliptic integrals of the first and second kind, respectively, and we have defined the three auxiliary functions

$$
\begin{aligned}
T & =\sqrt{F(1-\gamma) / \pi}, \\
V & =T^{2}+r^{2}(s)+\left(z\left(s_{N}\right)-z(s)\right)^{2}, \\
W & =2 \operatorname{Tr}(s) .
\end{aligned}
$$


The numerical solution now follows lines similar to those outlined by Forbes and Hocking [2]. An initial guess is made for the vector of unknowns

$$
\mathbf{u}=\left[z_{1} ; r_{2}^{\prime}, r_{3}^{\prime}, \ldots, r_{N}^{\prime}\right]^{\top},
$$

and using the conditions (2.20) at the top of the plume in the form

$$
r_{1}=0, \quad r_{1}^{\prime}=1, \quad z_{1}^{\prime}=0,
$$

all the other variables at the interface may be calculated. The arclength condition (2.19) gives

$$
z_{j}^{\prime}=-\sqrt{1-\left(r_{j}^{\prime}\right)^{2}} \quad j=2,3, \ldots, N,
$$

and the point values $r_{2}, r_{3}, \ldots, r_{N}$ and $z_{2}, z_{3}, \ldots, z_{N}$ representing the shape of the interface between the wetted and dry rock are obtained by exact integration of a cubic spline fitted through the values of $r_{j}^{\prime}$ and $z_{j}^{\prime}, j=1,2,3, \ldots, N$. Finally, the surface condition (2.10) gives $\phi_{j}=z_{j} / F, j=1,2,3, \ldots, N$.

The initial estimate (3.3) for the vector $u$ of unknowns is improved iteratively using Newton's method. The integral equation (2.21a) serves as an objective function, and the numerical task is therefore to make the residuals

$$
\begin{gathered}
E_{j}=\frac{1}{\sqrt{r_{j}^{2}+\left(z_{j}+1\right)^{2}}}-\frac{\gamma}{\sqrt{r_{j}^{2}+\left(z_{j}+1+\lambda\right)^{2}}} \\
-\int_{s_{1}}^{s_{N}}\left[\phi(\sigma)-\phi_{j}\right] \mathbb{K}(A, B, C, D) d \sigma-\mathbb{L}(T, V, W), \\
j=1,2,3, \ldots, N,
\end{gathered}
$$

tend to zero. The function $\mathbb{Q}$ is the contribution from the infinite tail of the integral, as in (3.2), and the integrals in (3.4) are performed by exact integration of a cubic spline fitted through the point values of the (nonsingular) integrand.

\section{Presentation of results for point source and sink}

We begin the discussion of the numerical results by repeating the remark made earlier in Section 2, namely, that the parameter $F$ could have been scaled out of the problem by adopting a different choice of dimensionless variables. Although this would have simplified the statement of the problem by reducing the number of parameters to two, it would have removed the opportunity to compute the maximum permissible volume inflow rate $Q_{1}$ before the top of the plume reaches ground level, and the ground thus becomes flooded with the leaching fluid. This problem is now addressed. 
The parameter $F$ can be scaled out of the problem by dividing all lengths and multiplying all pressures by the factor $\sqrt{F}$. Suppose that, for two different values $F_{1}$ and $F_{2}$ of this parameter, the top of the plume is computed to have heights $z^{(1)}(0)$ and $z^{(2)}(0)$ respectively. Then it follows that

$$
\frac{z^{(2)}(0)+1}{z^{(1)}(0)+1}=\sqrt{\frac{F_{2}}{F_{1}}} .
$$

Now let $F_{2}$ take the maximum permissible value $F_{\max }$, at which $z^{(2)}(0)=0$, corresponding to the point at which the top of the plume just reaches ground level. Equation (4.1) yields the formula

$$
F_{\max }=F_{1} /\left[z^{(1)}(0)+1\right]^{2},
$$

for the maximum value of $F$. In the absence of the sink, $\gamma=0$, we have computed ten solutions at different values of $F=F_{1}$, and recorded the maximum plume height $z^{(1)}(0)$ given directly by the Newton solution technique described in Section 3. On the basis of these results, (4.2) gives $F_{\max }=9.863$ to four significant figures accuracy, and so in dimensional variables

$$
Q_{1}<9.863 \rho g H^{2} D .
$$

The condition (4.3) could be regarded as an operating principle for the process of mineral leaching, in order to prevent surface flooding with leaching fluids, and the consequent damage to machinery and injury to personnel.

The influence of the point sink on the flow field is now considered. Figure 3 shows three interface profiles obtained with $F=0.2$ and separation distance $\lambda=0.1$, for the three values $\gamma=0,0.28$ and 0.8 of the relative withdrawal rate. In this diagram, the withdrawal point is vertically below the source; thus the source is at $z=-1$ and the sink is positioned at $z=-1.1$.

The profile obtained with $\gamma=0$ in Figure 3 represents the case in which the point sink is absent, and a vertical axi-symmetric wetted region is thus produced. As the withdrawal rate $\gamma$ is increased, the top of the plume remains at roughly the same height, but the plume radius decreases in accordance with (2.13) as required to conserve mass. The curve shown with a solid line corresponds to $\gamma=0.28$, and represents the maximum value of $\gamma$ at which we could compute a feasible solution satisfying the monotonicity requirement (2.23), for this value of the separation distance $\lambda$. During mineral leaching, it would presumably be desirable to operate as closely as possible to this optimum case $\gamma=0.28$, for the separation distance $\lambda=0.1$. Notice, however, that the loss rate is nevertheless about $72 \%$, which is very high and could be of economic and environmental concern.

Beyond the maximum withdrawal rate $\gamma=0.28$, the model breaks down, since the interface between the wetted and dry regions develops an overhanging portion, 


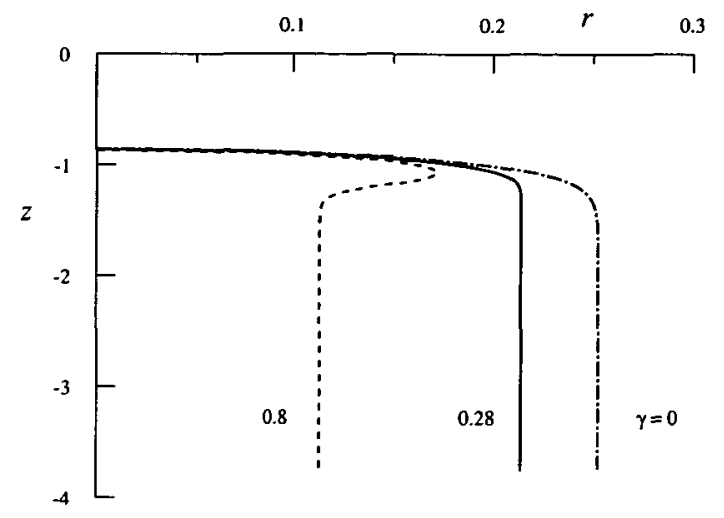

FIGURE 3. Three solutions obtained with recovery rates $\gamma=0$ (broken line), $\gamma=0.28$ (optimum case, solid line) and $\gamma=0.8$ (infeasible, dashed line), for the case $F=0.2, \lambda=0.1$.

violating the feasibility condition (2.23). Negative pressures are therefore generated in some regions within the wetted plume, and the assumption that the rock is fully saturated in these regions could no longer be maintained. A more complete model of the physical phenomenon in such cases would need to include the effects of partial saturation, and quite possibly also allow for unsteady flow, but these effects are not considered here. The numerical method of Section 3 nevertheless produces a solution profile, and an example of such an overhanging interface is given in Figure 3, for the withdrawal rate $\gamma=0.8$. The solution is sketched with a dashed line to indicate its lack of physical significance.

It is natural to ask whether better withdrawal rates $\gamma$ might be able to be achieved by placing the withdrawal point above the injection point, rather than below it as in Figure 3. This question is considered in Figure 4, in which the parameter $F$ again has the value $F=0.2$, and the separation parameter is now $\lambda=-0.04$. Thus the sink at $z=-0.96$ is now located directly above the source point at $z=-1$. Two profiles are shown in Figure 4, for withdrawal rates $\gamma=0$ and $\gamma=0.21$.

When the sink point lies above the source, it is again found that there is a maximum achievable recovery rate $\gamma$, but in this case the upper bound on $\gamma$ is a consequence of the failure of the numerical method, rather than a breakdown of the physical model. Thus the solution obtained with $\gamma=0.21$, sketched with a solid line in Figure 4, represents the largest withdrawal rate $\gamma$ for which our numerical method converged, when $\lambda=-0.04$. Solutions for larger $\gamma$ do not appear possible, yet the solution profile with $\gamma=0.21$ shown in Figure 4 gives no immediate clue as to the physical explanation for this upper bound on $\gamma$. Based on the work of Lucas, Blake and Kucera [7], we are prepared to suggest that, for $\gamma \approx 0.21$, a downward-pointing cusp 


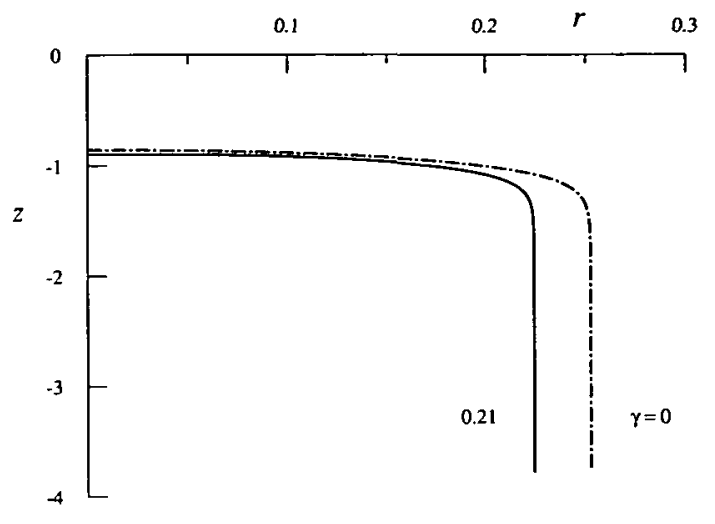

FIGURE 4. Two solutions obtained with recovery rates $\gamma=0$ (broken line) and $\gamma=0.21$ (maximum, solid line), for the case $F=0.2, \lambda=-0.04$.

might be formed at the top of the plume; if the withdrawal rate $\gamma$ were increased beyond this maximum value, then the cusp would be drawn rapidly down into the point sink, and in the actual leaching process, air would be recovered along with the leaching fluid. Cusped free surface profiles are also known in the literature on withdrawal from dams, for example, and the reader is referred to the paper by Tuck and Vanden-Broeck [11].

It is clear from Figure 4 that placing the sink above the source is not as efficient as locating it beneath the injection point. In Figure 3, a maximum feasible withdrawal rate $\gamma=0.28$ was achieved when the source and sink were a distance $|\lambda|=0.1$ apart, but when the sink is above the source in Figure 4, the maximum recovery rate is reduced to only $\gamma=0.21$, even for a much smaller separation distance $|\lambda|=0.04$ between the injection and recovery points. Of course, this is due to the fact that, by placing the sink below the source, the leaching process is aided by the effects of gravity, whereas in Figure 4 gravity is hindering the recovery of the leaching fluid.

Figure 5 represents a summary of the results obtained from about 160 separate converged solutions, computed with the numerical method of Section 3 for a variety of values of $\gamma$ and $\lambda$. For convenience, we have chosen $F=0.2$, but the parameter $F$ can be scaled out of these results by dividing the separation distance $\lambda$ on the horizontal axis by the quantity $\sqrt{F}$. Thus the results of Figure 5 present a rather complete statement of the parameter regions within which steady leaching with a fully wetted region of rock is possible. The circles on the graph indicate the maximum value of the withdrawal rate $\gamma$ that can be achieved for a fixed separation distance $\lambda$ between the source and the sink. Positive values of $\lambda$ indicate that the sink is below the source, and negative values correspond to the sink lying above the source. Leaching 


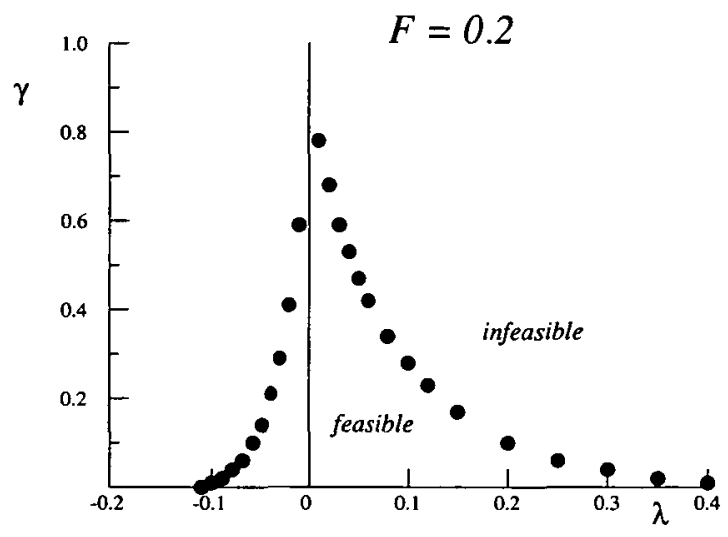

FIGURE 5. Feasible and infeasible solution regions in the $\lambda-\gamma$ parameter space, for $F=0.2$. The points represent the maximum feasible recovery rates $\gamma$.

is therefore possible in the parameter region enclosed by the points and the horizontal axis, but is infeasible outside this region. The results in Figure 5 clearly show the advantage of operating the process with the sink located beneath the source point.

\section{A point source and a ring sink}

An obvious question raised by the results in Figure 5 is whether it is possible to increase the recovery yield $\gamma$ of the leaching fluid using a different withdrawal geometry. This is, of course, an issue of great complexity, and cannot be fully answered here in the context of a relatively simple axi-symmetric flow, but the aim of the present section is to show that recovery rates $\gamma$ can indeed be enhanced by alternative leaching strategies, and so to provide a justification for further research on the topic.

We briefly consider here the effect of replacing the single point sink with a circular continuous line of sinks, at the same submergence depth $z=-1-\lambda$ and of overall relative withdrawal rate $\gamma$. The ring sink thus produced has dimensionless radius $\xi$. We stress the point that the use of a ring sink is purely to demonstrate that enhanced recovery rates $\gamma$ are possible, and to preserve the simple axisymmetric flow modelled here, and it is certainly not being suggested that such a method of fluid recovery should be considered as a serious in situ leaching strategy.

The only change required to the mathematical formulation derived in Section 2 is to the integral equation $(2.21 \mathrm{a})$, where the term $\gamma / \sqrt{r^{2}(s)+(z(s)+1+\lambda)^{2}}$ must be 


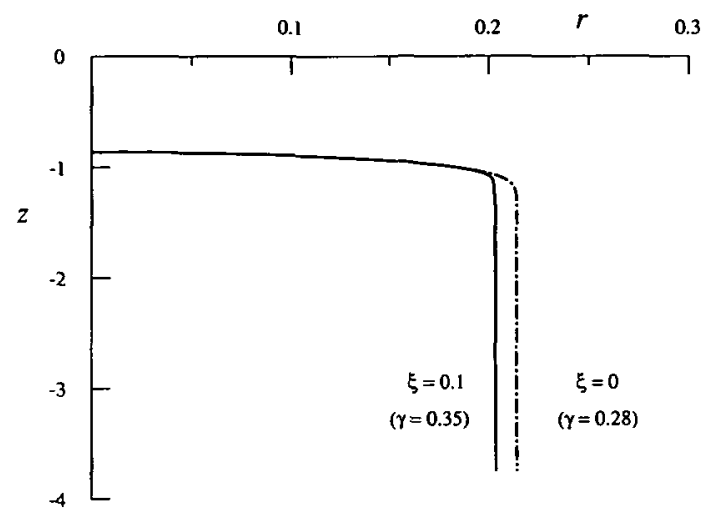

FIGURE 6. Comparison of the solutions at the maximum recovery rates for the case $F=0.2, \lambda=0.1$, for the point sink $(\xi=0, \gamma=0.28$, broken line $)$ and a ring sink of radius $\xi=0.1(\gamma=0.35$, solid line).

replaced by the quantity

$$
\frac{\gamma}{2 \pi} \int_{0}^{2 \pi} \frac{d \beta}{\sqrt{M-N \cos \bar{\beta}}}=\frac{2 \gamma}{\pi \sqrt{M+N}} K\left(\frac{2 N}{M+N}\right),
$$

in which the symbol $K$ again denotes a complete elliptic integral of the first kind, and the auxiliary functions $M$ and $N$ are defined to be

$$
M=r^{2}(s)+\xi^{2}+(z(s)+1+\lambda)^{2} \quad N=2 r(s) \xi .
$$

The numerical solution technique of Section 3 is again available for the solution of this problem.

When the radius $\xi$ of the ring sink is zero, the problem reduces to the previous case in which fluid recovery occurred through a point sink. However, as $\xi$ is increased, we find that the maximum recovery rate $\gamma$ also increases to some maximum. If the ring radius $\xi$ is increased still further, the maximum recovery rate $\gamma$ begins to decrease again, and eventually falls below the value which was achievable just for the simple point sink. This is because the ring sink disturbs the interface too violently if its radius is too large, but has little effect on the core of the wetted plume.

Figure 6 shows the type of improvement in the maximum recovery rate $\gamma$ that is typically possible when the ring radius $\xi$ is near to its optimum value. In this case, $F=0.2$ and $\lambda=0.1$, and the profile shown for radius $\xi=0(\gamma=0.28)$ corresponds to the maximum feasible withdrawal rate for a point sink, as shown in Figure 3. However, when the radius is increased to $\xi=0.1$, the maximum recovery rate increases to $\gamma=0.35$, and this profile is sketched with a solid line in Figure 6. 
This represents an increase of about $25 \%$, and this is typical of other cases in which we have compared the effects of the ring sink with the point sink recovery point.

\section{Discussion}

In this paper we have discussed a simple model of the process of in situ mineral leaching, in which a caustic leaching fluid is introduced into a homogeneous rock at one point, and collected at another point either vertically above or below the injection point. The region of rock that is wetted by the fluid forms a vertical plume down to infinite submergence depth, and the main aim of this preliminary study has been to determine the proportion of leaching fluid that can be recovered from the rock. The results presented in Figure 5 show that the maximum fraction of the fluid that can be recovered is critically dependent upon the separation distance between the injection point and the recovery point. If the two points are very close together $(\lambda \rightarrow 0)$, then it is possible to reclaim a high percentage of the original leaching fluid. However, only a small region of rock would be inundated, and so the amount of mineral that could be recovered would be rather small. By contrast, if the separation distance between the injection and withdrawal points is large, then a large volume of rock could be leached, but only a small percentage of the mineral-bearing fluid could be retrieved, with consequent high loss rates. This might be of environmental concern. In this way, there is an effective trade-off between the volume of rock between the two points that can be reached by the leaching fluid, and the amount that can be recovered.

We investigated an alternative recovery strategy for the fluid, and demonstrated an improvement of about $25 \%$ in the maximum withdrawal rate of the fluid from the rock. A rather unrealistic geometry was chosen, motivated chiefly by the desire to retain axi-symmetric geometry and thus the problem formulation of Section 2 and the solution technique of Section 3. Nevertheless, the improvements in recovery fraction were sufficiently great to justify a more detailed investigation of alternative leaching strategies. The present integral-equation formulation is certainly capable of coping with an arbitrary arrangement of source and sink points, as would presumably occur on an actual mining site, but the axi-symmetric geometry assumed here would of course be lost, and solutions would only be obtained at a very considerable increase in computing time.

Our integral-equation approach can, to a certain extent, cope with the presence of strata within the rock. However, if the non-uniformity of rock properties is more generalized, boundary element methods of solution are not appropriate, and a finitedifference or finite-element approach would be needed.

The real restriction on our ability to reclaim the leaching fluid from the rock comes from the monotonicity condition (2.23), which states that overhanging portions of the 
interface are not possible in our model. (If they occurred, negative pressures would appear within the wetted region.) In principle, this restriction could be overcome if the rock outside the region inundated with leaching fluid were not dry, as assumed here, but were instead saturated with water. (We are indebted to Dr Alex McNabb for suggesting this possibility.) The leaching strategy would then be somewhat similar to the secondary recovery of oil from oil-wells, and greatly increased mineral recovery rates should be possible. We are presently investigating this two-phase flow.

\section{References}

[1] L. K. Forbes, "On the effects of nonlinearity in free-surface flow about a submerged point vortex", J. Engineering Maths. 19 (1985) 139-155.

[2] L. K. Forbes and G. C. Hocking, "Flow caused by a point sink in a fluid having a free surface", $J$. Austral. Math. Soc. Ser. B 32 (1990) 231-249.

[3] M. J. Friedel, "Modeling in situ copper leaching in an unsaturated setting", Bureau of Mines Report 9386, U. S. Dept. of the Interior, 1991.

[4] G. C. Hocking, "Cusp-like free-surface flows due to a submerged source or sink in the presence of a flat or sloping bottom", J. Austral. Math. Soc. Ser. B 26 (1985) 470-486.

[5] L. Landweber and M. Macagno, "Irrotational flow about ship forms", lowa Inst. of Hydraulic Res. Rep. IIHR 123 (1969).

[6] H. Levine and Y. Yang, "A rising bubble in a tube", Phys. Fluids A 2 (1990) 542-546.

[7] S. K. Lucas, J. R. Blake and A. Kucera, "A boundary-integral method applied to water coning in oil reservoirs", J. Austral. Math. Soc. Ser. B 32 (1991) 261-283.

[8] M. Miksis, J. M. Vanden-Broeck and J. B. Keller, "Axisymmetric bubble or drop in a uniform flow", J. Fluid Mech. 108 (1981) 89-100.

[9] R. Schmidt, K. C. Behnke and M. J. Friedel. "Hydrologic considerations of underground in situ copper leaching", Soc. Min. Eng. AIME, 1990, preprint.

[10] O. D. L. Strack, Groundwater Mechanics (Prentice Hall, Englewood Cliffs, N. J., 1989).

[11] E. O. Tuck and J. M. Vanden-Broeck, "A cusp-like free-surface flow due to a submerged source or sink", J. Austral. Math. Soc. Ser. B 25 (1984) 443-450. 